\title{
Measurement of Inclusive Quasielastic Scattering of Polarized Electrons from Polarized ${ }^{3} \mathrm{He}$
}

\author{
C. E. Woodward, E. J. Beise, J. E. Belz, R. W. Carr, B. W. Filippone, W. B. Lorenzon, R. D. McKeown, \\ B. Mueller, and T. G. O'Neill \\ W. K. Kellogg Radiation Laboratory, California Institute of Technology, Pasadena, California 91125 \\ G. Dodson, K. Dow, M. Farkhondeh, S. Kowalski, K. Lee, N. Makins, R. Milner, A. Thompson, D. Tieger, \\ J. van den Brand, A. Young, X. Yu, and J. D. Zumbro \\ Bates Linear Accelerator Center, Laboratory for Nuclear Science and Department of Physics, \\ Massachusetts Institute of Technology, Cambridge, Massachusetts 02139 \\ (Received 7 May 1990; revised manuscript received 18 June 1990)
}

\begin{abstract}
We report a measurement of the asymmetry in spin-dependent quasielastic scattering of longitudinally polarized electrons from a polarized ${ }^{3} \mathrm{He}$ gas target. This measurement represents the first demonstration of a new method for studying electromagnetic nuclear structure: the scattering of polarized electrons from a polarized nuclear target. The measured asymmetry is in good agreement with a Faddeev calculation and supports the picture of spin-dependent quasielastic scattering from polarized ${ }^{3} \mathrm{He}$ as predominantly scattering from a polarized neutron.
\end{abstract}

PACS numbers: $24.70 .+\mathrm{s}, 25.10 .+\mathrm{s}, 25.30 . \mathrm{Fj}, 29.25 . \mathrm{Kf}$

An important method of investigating nuclear structure and dynamics is the use of elastic and inelastic electron scattering from nuclei. In such studies, one probes the electromagnetic structure of the nucleus, which may be characterized by matrix elements of the electromagnetic current operators. Particularly for few-nucleon systems, this allows a relatively direct comparison of experimental data with the results of detailed theoretical calculations. For the case of inclusive electron scattering from unpolarized nuclei, it is only possible to extract two response functions from the differential scattering cross section; these correspond to the longitudinal and transverse components of the nuclear current. This is the usual Rosenbluth separation for inclusive electron scattering, ${ }^{1}$ which requires varying the kinematics of the incident and scattered electrons while keeping the energy and three-momentum transfer fixed. In general, the longitudinal response involves the sum of the squares of the charge multipole matrix elements, while the transverse response contains the sum of the squares of the electric and magnetic multipole matrix elements which are permitted by conservation of angular momentum, parity, and time-reversal invariance. Since a complete understanding of the electromagnetic structure of the nucleus requires a knowledge of all of the individual matrix elements, and since each response function generally contains a number of different matrix elements, in general, it is not possible to extract all of the individual terms from such experiments. With polarized beam and target it is possible to perform a generalized version of the Rosenbluth separation of the inclusive cross section into additional response functions. ${ }^{2}$ In this way additional nuclear-structure information may be obtained from inclusive spin-dependent electron scattering. As a result, new experimental programs utilizing polarized beams and polarized targets are being planned for all major electron accelerator laboratories (SLAC, MIT-Bates, CEBAF, Mainz, Bonn, DESY HERA, and Novosibirsk).

${ }^{3} \mathrm{He}$ is a particularly interesting nucleus with which to carry out the generalized Rosenbluth separation. It is anticipated that spin-dependent quasielastic scattering from ${ }^{3} \mathrm{He}$ should be sensitive to the neutron electromagnetic form factors. Since the nucleus in its ground state is predominantly a spatially symmetric $S$ state, the two protons are mainly in a spin singlet state. Further, the three-body system has the important advantage that realistic calculations can be carried out. In fact, a calculation using Faddeev wave functions by Blankleider and Woloshyn ${ }^{3}$ has demonstrated that, in the vicinity of the quasielastic peak, spin-dependent effects should be determined primarily by scattering from the neutron. The differential cross section for inclusive spin-dependent scattering may be written as ${ }^{2}$

$$
\frac{d \sigma}{d \Omega d E^{\prime}}=\Sigma \pm \Delta\left(\theta^{*}, \phi^{*}\right)
$$

where the $+(-)$ sign corresponds to right (left) helicity state for the incident electron. The asymmetry $A$ is the ratio of spin-dependent to unpolarized cross sections,

$$
A=\Delta\left(\theta^{*}, \phi^{*}\right) / \Sigma \text {, }
$$

and the experimental asymmetry $A_{\text {expt }}$ is related to $A$ by

$$
A_{\text {expt }}=p_{e} p_{T} A \text {, }
$$

where $p_{e}$ and $p_{T}$ are the electron and target polarizations, respectively. The direction of the target polarization is specified by the angles $\theta^{*}$ and $\phi^{*}$, as defined in Fig. 1. Further, for ${ }^{3} \mathrm{He}$, a spin- $\frac{1}{2}$ target, the asym- 


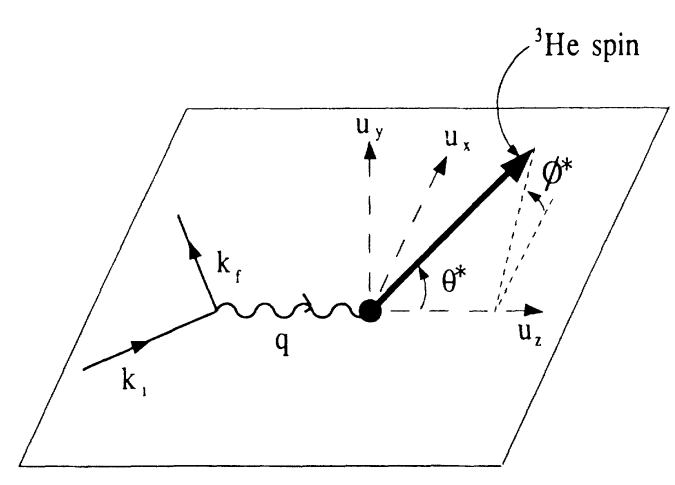

FIG. 1. Kinematics for electron scattering from polarized targets. Here $\mathbf{u}_{z}$ is along the direction of momentum transfer q. The vector $\mathbf{u}_{y}$ is normal to the electron-scattering plane and $\mathbf{u}_{x}=\mathbf{u}_{y} \times \mathbf{u}_{z}$ lies in the scattering plane. The target polarization direction is then specified by the angles $\left(\theta^{*}, \phi^{*}\right)$ in this coordinate system.

metry is given by ${ }^{2}$

$$
A=-\frac{\cos \theta^{*} v_{T^{\prime}} R_{T^{\prime}}+2 \sin \theta^{*} \cos \phi^{*} v_{T L^{\prime}} R_{T L^{\prime}}}{v_{L} R_{L}+v_{T} R_{T}}
$$

where the $v_{K}$ are kinematic factors (see Ref. 2). $R_{L}\left(Q^{2}, \omega\right)$ and $R_{T}\left(Q^{2}, \omega\right)$ are the longitudinal and transverse response functions contributing to the unpolarized cross section and are functions of the square of the momentum transfer $Q^{2}$ and the electron energy loss $\omega$. $R_{T^{\prime}}\left(Q^{2}, \omega\right)$ and $R_{T L^{\prime}}\left(Q^{2}, \omega\right)$ are two new response functions that do not contribute to the unpolarized inclusive cross section. $R_{T^{\prime}}$ is a transverse response function whereas $R_{T L^{\prime}}$ arises from interference of transverse and longitudinal multipoles. By orientation of the target spin at $\theta^{*}=0^{\circ}$ (along q) or at $\theta^{*}=90^{\circ}$ (normal to q), one can select the resulting asymmetry to be proportional to $R_{T^{\prime}}$ or $R_{T L^{\prime}}$, respectively. Assuming the impulse approximation (ignoring final-state interactions and meson-exchange currents) the calculations of Blankleider and Woloshyn ${ }^{3}$ indicate that $R_{T^{\prime}}$ is predominantly proportional to the square of the neutron magnetic form factor $\left(G_{M}^{n}\right)^{2}$ and $R_{T L^{\prime}}$ is very sensitive to the product $G_{E}^{n} G_{M}^{n}$. Experimental confirmation of this picture would give important motivation for future experiments while deviations from the simple picture would provide new information on the physics of the three-nucleon system. The dependence of $R_{T L^{\prime}}$ on the neutron electric form factor is particularly interesting since this form factor is poorly determined experimentally.

The measurement reported in this Letter was performed at quasielastic kinematics near $\theta^{*}=0^{\circ}$ where the response function $R_{T^{\prime}}$ dominates the spin-dependent cross section. The experiment was carried out at the MIT-Bates Linear Accelerator Center using a polarized electron beam at $574-\mathrm{MeV}$ incident energy. This value was chosen for the experiment because the $g-2$ precession of an electron extracted from the source after ac- celeration and transport into the experimental hall is $2 \pi$ rad. Thus, electrons with longitudinal polarization extracted from the polarized electron source have longitudinal polarization at the target. The source of polarized electrons was a crystal of GaAs optically pumped by a $\mathrm{Kr}$-ion laser. The average current on the target during the experiment was typically $10 \mu \mathrm{A}$. The average electron polarization was determined to be $0.41 \pm 0.04$ by measurement of Møller scattering from a removable magnetized foil upstream of the target. ${ }^{4}$

To carry out the experiment a new type of polarized gas target was developed; a detailed description will be reported elsewhere. ${ }^{5}$ The ${ }^{3} \mathrm{He}$ was polarized through metastability exchange optical pumping. ${ }^{6}$ The target was built as a double-cell system and consisted of a Pyrex optical pumping cell at room temperature connected by a Pyrex transfer tube to a copper target cell which was cooled to $17 \mathrm{~K}$. The ${ }^{3} \mathrm{He}$ gas pressure was 2 Torr. The metastable population was created in the pumping cell by maintaining a weak discharge. The optical pumping light was supplied by a Nd-doped yttrium aluminum garnet crystal pumped by a krypton arc lamp in a Lasermetrics 9650 cavity. The target cell was 16 $\mathrm{cm}$ long, $2.54 \mathrm{~cm}$ wide, and had $0.025-\mathrm{cm}$-thick walls. The entrance and exit foil windows were $5-\mu \mathrm{m}$-thick copper. The target walls were coated with a thin layer of $\mathrm{N}_{2}$ to reduce surface-induced spin relaxation at low temperatures. The spins were aligned in a holding field of approximately $30 \mathrm{G}$ provided by Helmholtz coils which surrounded the target system. The polarization of the ${ }^{3} \mathrm{He}$ in the pumping cell was monitored continuously by detection of the circular polarization of the $667-\mathrm{nm}$ line excited by the discharge. The target cell polarization was inferred from the polarization of the pumping cell and the time constants of the coupled system. The target polarization during the experiment typically ranged between $20 \%$ and $30 \%$, depending primarily upon the laser output power and the electron-beam intensity. The presence of the electron beam reduced the target polarization to typically $85 \%$ of the value when the beam was off.

Electrons scattered from the polarized ${ }^{3} \mathrm{He}$ target were detected at quasielastic kinematics in the OHIPS magnetic spectrometer. The spectrometer was configured at an angle of $51.1^{\circ}$ and a central momentum of $462 \mathrm{MeV} / c$ for an average four-momentum transfer $Q^{2}$ of $0.2(\mathrm{GeV} / c)^{2}$. Collimators were used to limit the target thickness viewed by the spectrometer to $10 \mathrm{~cm}$. With no gas in the target the rate in the spectrometer was less than $10 \%$ of the rate with the target full. Since the polarized ${ }^{3} \mathrm{He}$ target atoms are present as pure atomic species, the only unpolarized material contributing to the observed yield was the target cell itself and so was accounted for as an $8 \%$ reduction in the experimentally measured asymmetry. The beam position was continuously monitored for helicity correlated shifts that could have caused systematic asymmetries due to the target walls; the fractional effect on the measured ${ }^{3} \mathrm{He}$ asym- 
TABLE I. Results of asymmetry measurements.

\begin{tabular}{lrrc}
\hline \hline $\begin{array}{c}\text { Charge } \\
(\mu \mathrm{A} \mathrm{h})\end{array}$ & $\begin{array}{c}\theta^{*} \\
(\mathrm{deg})\end{array}$ & $\begin{array}{c}\phi^{*} \\
(\mathrm{deg})\end{array}$ & \multicolumn{1}{c}{$\begin{array}{c}A \\
(\%)\end{array}$} \\
\hline 310 & 0.9 & 180 & $-3.5 \pm 2.4$ \\
342 & 7.9 & 180 & $-2.1 \pm 2.7$ \\
826 & 172.1 & 0 & $+4.2 \pm 1.7$ \\
& & & $-3.49 \pm 1.23 \pm 0.54$ \\
1478 (combined) & & & $-4.3 \pm 0.2$ \\
Theory (Ref. 3) & & & $-4.5 \pm 0.2$ \\
Theory (Ref. 8) & & &
\end{tabular}

metry was less than $0.5 \%$. The unpolarized ${ }^{3} \mathrm{He}$ quasielastic cross section was found to be in good agreement $(\sim 10 \%)$ with previous data, ${ }^{7}$ and served as a continuous check on beam halo and target-wall scattering.

The scattering asymmetry was measured for three different configurations of target spin direction and the results are given in Table I. A total of $1478 \mu \mathrm{A} \mathrm{h}$ of charge was collected. Corrections were applied to the data to account for radiative corrections $[(1.8 \pm 0.7) \%$ of $A]$ and pion contamination [ $(3 \pm 3) \%$ of $A]$. Additional significant sources of systematic error were in the determination of the beam polarization $( \pm 10 \%)$, the target polarization ( $\pm 10 \%)$, and the empty-target contribution $( \pm 2 \%)$. All other systematic errors are estimated to be negligible in comparison. Combining all the data (reversing the sign of the $\theta^{*}=172.1^{\circ}$ result) yields the asymmetry $A=(-3.49 \pm 1.23 \pm 0.54) \%$, where the first uncertainty is statistical and the second is the systematic uncertainty. The calculation of Blankleider and Woloshyn has been averaged over the spectrometer acceptance at these kinematics to yield the prediction $A=(-4.3 \pm 0.2) \%$. A similar calculation based on Faddeev wave functions from Friar ${ }^{8}$ gives the prediction $A=(-4.5 \pm 0.2) \%$. Thus, the results of the experiment are well described by the conventional model.

The small uncertainties in the theoretical asymmetries arise from nuclear-structure uncertainties and the uncertainty associated with averaging over the experimental acceptance. Thus, a more precise measurement of the asymmetry $(\sim 10 \%)$ could be used to extract a value of the neutron magnetic form factor in ${ }^{3} \mathrm{He}$ with about $5 \%$ precision. (Only $26 \%$ of the asymmetry arises from the protons in ${ }^{3} \mathrm{He}$.) Sufficient statistical precision could be attained with about $6 \mathrm{~d}$ of running with higher $(40 \mu \mathrm{A})$ beam current. Work is in progress to reduce the systematic errors associated with beam and target polarizations.

In summary, the first measurement of a spin-dependent asymmetry in the scattering of polarized electrons from a polarized nuclear target has been performed. This result demonstrates a powerful new technique in electromagnetic nuclear physics. The experiment was feasible because of the newly developed polarized gas target. The measured asymmetry supports the conclusion that spin-dependent quasielastic scattering from polarized ${ }^{3} \mathrm{He}$ is predominantly due to scattering from a polarized neutron. These results provide strong motivation to proceed with further experiments on spindependent electron scattering from ${ }^{3} \mathrm{He}$.

We would like to thank B. Blankleider and $R$. Woloshyn for providing us with their computer code. We also acknowledge the assistance of $T$. Gentile in checking the sign of the target polarization. The research has been supported by the National Science Foundation Grant No. PHY88-17296 (Caltech) and by the U.S. Department of Energy Contract No. DEAC02-76ER03069 (MIT).

${ }^{1}$ M. N. Rosenbluth, Phys. Rev. 79, 615 (1950).

${ }^{2}$ T. W. Donnelly and A. S. Raskin, Ann. Phys. (N.Y.) 169, 247 (1986).

${ }^{3}$ B. Blankleider and R. M. Woloshyn, Phys. Rev. C 29, 538 (1984).

${ }^{4}$ E. J. Beise et al. (to be published).

${ }^{5}$ R. G. Milner, R. D. McKeown, and C. E. Woodward, Nucl. Instrum. Methods Phys. Res., Sec. A 274, 56 (1989); C. E. Woodward et al. (to be published).

${ }^{6}$ F. D. Colegrove, L. D. Shearer, and G. K. Walters, Phys. Rev. 132, 2561 (1963); M. Leduc et al., Nucl. Sci. Appl. 1, 1 (1983); C. L. Bohler et al., J. Appl. Phys. 63, 2497 (1988).

${ }^{7}$ K. Dow et al., Phys. Rev. Lett. 61, 1706 (1988); K. Dow, Ph.D. thesis, MIT, 1987 (unpublished).

8 J. Friar (private communication). 